\title{
Original Research \\ Can hysteroscopy be substituted to hysterosalpingography in the assessment of infertility? Methods of showing tubal transition as hysteroscopic
}

\author{
Buket Aydın Yanar ${ }^{1}$, Eren Pek ${ }^{1, *}$, Mesut A. Unsal ${ }^{1}$ \\ ${ }^{1}$ Department of Obstetrics and Gynecology, Canakkale Onsekiz Mart University, 17020 Canakkale, Turkey \\ *Correspondence: drerenpek@hotmail.com (Eren Pek) \\ Academic Editor: Marco Noventa \\ Submitted: 17 January 2021 Revised: 11 May 2021 Accepted: 30 July 2021 Published: 12 January 2022
}

\begin{abstract}
Background: The main goal of this study was to prove that hysteroscopy is a superior method compared to hysterosalpingography in the evaluation of tubal passage and the uterine cavity in infertile women. Methods: The study was carried out on 30 volunteer women for whom evaluation of the uterine cavity and transit through the tubules was required due to infertility. In the evaluation of the hysteroscopic tubal passage, a $6 \mathrm{Fr}$ feeding cannula was advanced from the hysteroscope barrel, and firstly methylene blue and then an air bubble were applied to the fluid-filled uterine cavity through this flexible cannula. Results: When the reference method was taken as hysterosalpingography, the specificity of hysteroscopy was found to be $85.71 \%$ (95\% CI (confidence interval): $42.13 \%-$ 99.64\%), sensitivity $94.74 \%$ (95\% CI: $85.38-98.90 \%)$. The positive predictive value of hysteroscopy was calculated as $98.18 \%(95 \%$ CI: $89.78 \%-99.70 \%$ ) and the negative predictive value was $66.67 \%$ (95\% CI: 38.96\%-86.24\%). Observing the bubble and swirl effect together in the evaluation of the tube opening increases the diagnostic accuracy. And benefit of hysteroscopy in the evaluation of tubal passage was statistically significantly higher than hysterosalpingography. Conclusion: Considering the cellular damages that can be caused by hysterosalpingraphy and the real observation power provided by hysteroscopy, simultaneous evaluation and the comfort of making intervention possible, hysteroscopy will be a more useful and useful application.
\end{abstract}

Keywords: Hysteroscopy; Hysterosalpingography; Shawki-bubble test; Methylene blue test; Tubal passage

\section{Introduction}

Infertility is defined as the inability of a sexually active couple with no contraception to achieve pregnancy within one year [1]. The most important step in evaluating infertile couples and making treatment plans is to determine the etiology correctly. Tubal factors constitute $20-25 \%$ of the causes of infertility in women [2]. The fallopian tubes are serve a highly specialised essential functions in the reproductive process. The first mission, picking up an ovum when it is discharged from the ruptured ovarian follicle and transferring the ovum into the tubal lumen. The second mission of the tubes are, they provide a suitable environment for sperm capacitation and ovum fertilisation to occur, and transfers the fertilised embryo into the uterus [3].

Chromopertubation, in which hysteroscopy (HS) and laparoscopy are combined, is considered the gold standard method in evaluating the tube opening and uterine cavity [4-6]. However, research is still ongoing on an easy and simple method that can be applied in less invasive and outpatient settings. Today, hysterosalpingography (HSG), which can be used in an out-patient setting, is the most commonly used screening method for evaluation of the fallopian tubes [7]. However, recent studies have shown hysterosalpingography to exhibit a relatively low sensitivity to detect tubal patency and a high inter-observer variation in the interpretation of results [8]. In this context, studies on hysteroscopic methods are concentrated because they can simultaneously provide treatment in case of detection of pathological conditions of the uterine cavity. Undoubtedly, the most important reasons are that they are less invasive and less cost-effective [9-11]. In this study, we aimed to compare the traditionally applied hysterosalpingography in the evaluation of infertile couples and office hysteroscopy, which we think may be superior in many ways. The first is to evaluate the diagnostic capacity of hysteroscopy to evaluate tube patency compared to hysterosalpingography. Our second aim was to evaluate the uterine cavity simultaneously and to compare the results.

\section{Material and method}

\subsection{Ethics}

Date and number of approval obtained from Çanakkale Onsekiz Mart University Scientific Research Ethics Committee: 15.11.2017 / 2017-18 (2011-KAEK27/2017-E.98469).

\subsection{Sample}

The study was conducted on 30 female patients aged 18-40 years who were admitted to the Çanakkale Onsekiz Mart University hospital gynecology outpatient clinic of be- 
tween 2018 and 2019 due to primary or secondary infertility. The cases were evaluated prospectively.

\subsection{Exclusion criteria}

Women under the age of 18 , those who did not want to participate in the study, and those with previous uterine surgery were not included in the sample. Women with diagnosed chromosomal abnormalities that could cause uterine and ovarian anomalies were excluded from the study. Apart from these, chromosomal disorders that may cause thrombophilia and similar conditions, those with chronic diseases (such as diabetes, thyroid hormone disorders, prolactin hormone disorders) were not included in the study due to the possibility of tubal patency being affected. Except for these cases, no exclusion criteria were used.

\subsection{Processing algorithm}

Sociodemographic characteristics and medical history of the patients to be included in the study were obtained first, and then gynecological examinations were performed. The results of the ultrasonographic evaluation were not presented as they would not have any positive or negative effect on the purpose of the study. All hysterosalpingographic and hysteroscopic procedures were performed by the same physician. While planning the methodology of the study, a detailed literature search was conducted and an evaluation procedure suitable for our study was determined [10,12]. Both processes were performed during the late proliferative phase (10th day of menstruation). The evaluation of all results was first made by the same physician during the procedures. Then, images recorded in digital media by another physician were evaluated. Evaluation results of both physicians were the same. All procedures were performed consecutively under general anesthesia simultaneously on the same day. Since hysterosalpingography can cause spasms due to jet injection and open possible obstructions with pressure, the order of application of the procedures was as given on the side: (1) bubble-forming hysteroscopy, (2) application of methylene blue during hysteroscopy, and (3) hysterosalpingographic evaluation. Hysteroscopic evaluation was performed using $5 \mathrm{~mm}$ hysteroscop (Karl-Storz Endoscope) under conditions where distension medium was provided with saline. While evaluating the tubes hysteroscopically, methylene blue $(10 \%, 0.2 \mathrm{~mL})$ and bubbles were applied to near the tube openings through a $6 \operatorname{Fr}(2 \mathrm{~mm})$ flexible cannula passed through the hysteroscope channel. On the other hand, water-soluble radiopaque material (Omnipaque $300 \mathrm{mg} / \mathrm{mL}$, Opakim, Istanbul) was used in all women during HSG. And duly, radio-opaque medium was injected through the rubin cannula. The spread of this applied substance in the uterine cavity and its distribution to the abdominal cavity through the tubes were simultaneously monitored under fluoroscopy. And it was recorded digitally.

First, an evaluation of the uterine cavity for at least ten seconds was performed to allow pressure compensation before assessing the effect of air bubbling. Then the bubble evaluation phase was started. Approximately $0.25 \mathrm{~mL}$ of air was given very close to the tubal opening with the aid of a syringe attached to the cannula and large bubbles were formed in the uterine cavity. The observation of the passage of these large bubbles from the tube openings to the abdominal cavity was evaluated as an indicator for tubal patency. In contrast, if there is only one small $(<2 \mathrm{~mm})$ air bubble entering the ostium, if the air bubbles only entered before pressure equalization was finished, or if air bubbles were accumulating in the uterine cavity but could not pass through the tubal opening, this was not considered an indicator of tube patency. If the air bubbles did not pass through the ostia rapidly, tubal ostia were observed for 40 to $60 \mathrm{sec}-$ onds, considering the presence of possible temporary tubal spasms (parryscope technique) [12] (Fig. 1a,b). All these procedures were repeated separately for both tubal ostia.

Secondly, rhythmic movements of the tubal ostium and tubal patency were evaluated with highly concentrated methylene blue's transport. Each tubal ostium was observed for at least three minutes to assess the transport of the dye. The transition of methylene blue from the tubes to the abdominal cavity by forming a vortex, not accumulating in the uterine cavity, was interpreted as the presence of a tubal patency. However, the dilution and invisible distribution of methylene blue in the uterine cavity was interpreted as the absence of tubal patency (Fig. 2).

\subsection{Statistical analysis}

SPSS-20 (Statistical Package for the Social Sciences, Statistics Program for Social Sciences, Chicago, IL, USA) program was used for statistical analysis of the data obtained. The existence of statistical significance was evaluated by Fisher Exact test, and cases where the $p$ value was found to be less than 0.01 was considered statistically significant.

\section{Results}

The study was completed with the evaluation of a total of 30 women. In other words, 30 uterine cavities and 60 fallopian tubes were evaluated during the whole study. The women included in the study were women who consecutively applied to the outpatient clinic due to infertility and agreed to participate. In the ultrasonography performed on the third day of menstruation, no pathological sonographic finding was detected in 16 women $(53.3 \%)$. On the other hand, an appearance compatible with polyp was observed in sonography of two women (6.7\%), and an image compatible with uterine septum or arcuate uterus was observed in ultrasonography of five women (16.7\%). A clear diagnostic report on the uterine cavity could not be made for seven cases $(23.3 \%)$. When the uterine cavities of women were evaluated by hysterosalpingography, no uterine pathology was observed in 17 (56.7\%) women. On the other hand, fill- 


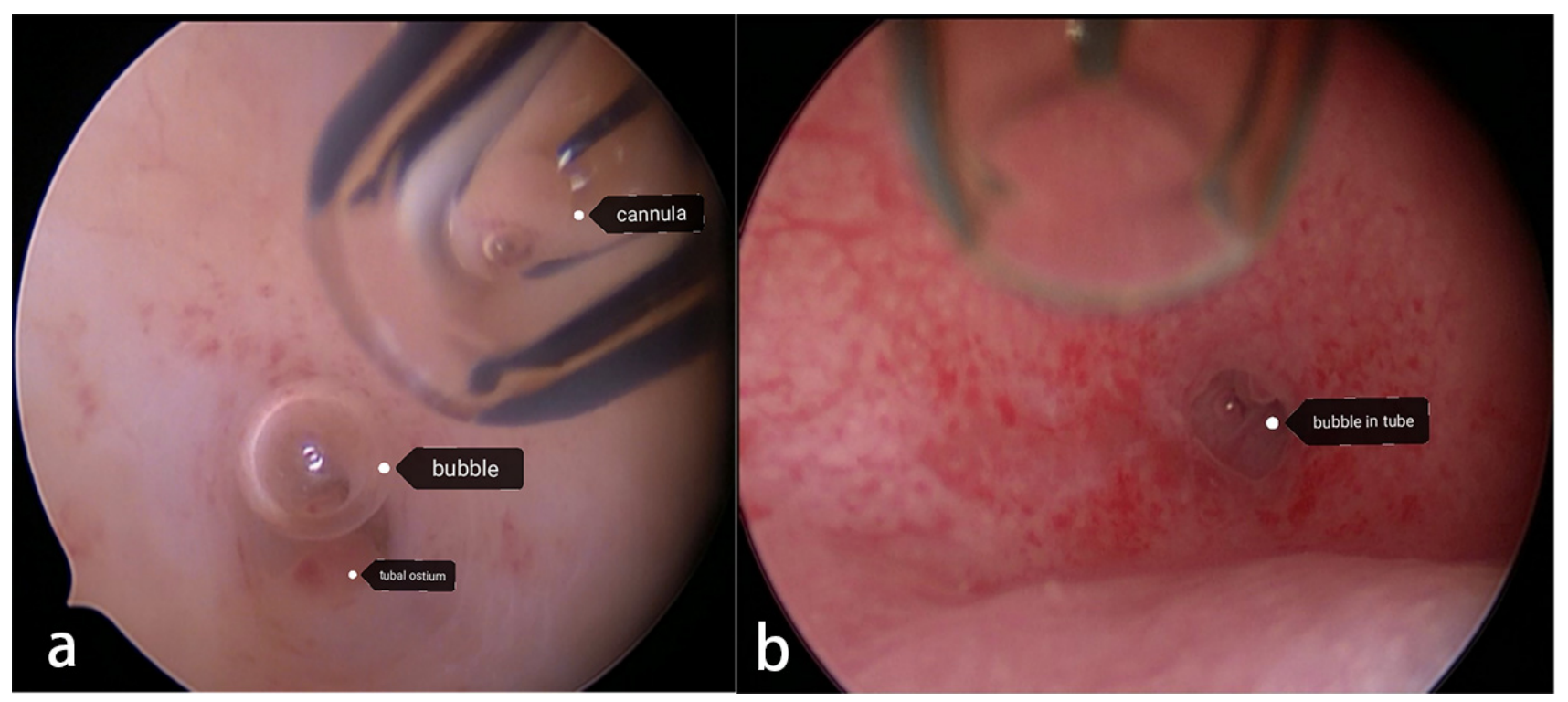

Fig. 1. Escape of the bubble from the tubal opening into the abdominal cavity.

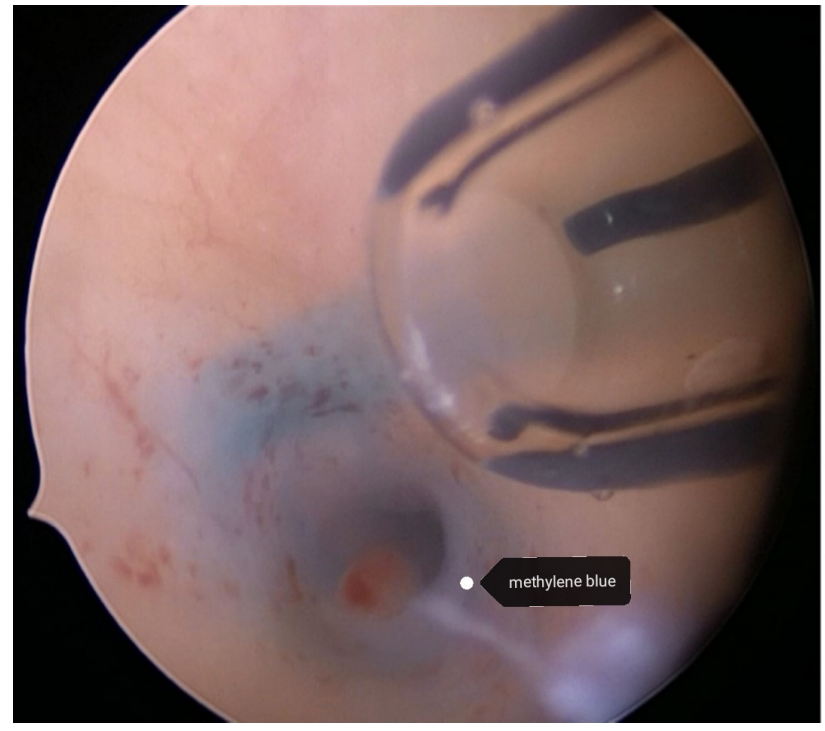

Fig. 2. Injection of methylene blue into the internal fallopian tube ostia and and passage of methylene blue from the tube into the abdominal cavity with the swirling effect.

ing defects (polyps) were found in the uterine cavity in two $(6.7 \%)$ women, and uterine septum or arcuate uterus was observed in nine (30\%) women. In addition, unfortunately, the uterine cavity could not be evaluated sufficiently in the images of two $(6.7 \%)$ women. As a result of the hysteroscopic evaluation of the cavities, no uterine pathology was observed in 17 (56.7\%) women; on the other hand, uterine septum or arcuate uterus was observed in eight $(26.7 \%)$ women, and endometrial polyp was found in five (16.7\%) women. All the comparative results are presented in Table 1 .

Only eleven of the seventeen women in whom the uterine cavity was reported as normal on hysterosalpingog- raphy and hysteroscopy had common findings in both, and the uterine cavities were normal. Filling defect was observed on hysteroscopy in four of the remaining six of the seventeen women in whom hysterosalpingography was reported to be normal. The septum or arcuate uterus was observed in two of the women who were reported as normal on hysterosalpingography. Although filling defects were found in two women in hysterosalpingography, none of these women had polypoid formation that could cause filling defect in hysteroscopy. However, polyps were detected in five women on hysteroscopy. And none of these women had polyps detected on hysterosalpingography. The hysterosalpingography of four women who were found to have polyps on hysteroscopy were evaluated as normal. It was evaluated that the remaining one might have a septum defect. In hysterosalpingography, it was thought that nine women might have a uterine septum or an arcuate uterus. However, in the hysteroscopic evaluations of these women, it was observed that two of them were normal and one of them had polyps. The septum was detected in only six of them by both methods. The hysteroscopic evaluation of two women whose hysterosalpingography could not be evaluated clearly was normal. All findings of the uterine cavity are presented in Table 2.

The evaluation of the tubes was performed separately for each woman as right and as a result of the evaluation of the tubes by hysteroscopic bubble (parryscope) and methylene blue methods, it was observed that there was a passage through the inner mouth of both fallopian tubes in twentytwo women. It was observed that three of the remaining eight women had no passage through the right tube and five of them through the left tube. In all cases where bubble transition was observed, the transition of methylene blue was also present. Likewise, in all cases where methylene blue passed, bubble transition occurred. As a result of the hys- 
Table 1. Ultrasonographic, hysterosalpingographic and hysteroscopic results of uterine findings.

\begin{tabular}{lccc}
\hline & Ultrasonography & Hysterosalpingography & Hysteroscopy \\
\hline Normal & $16(53.3 \%)$ & $17(56.7 \%)$ & $17(56.7 \%)$ \\
Filling defect (polyp) & $2(6.7 \%)$ & $2(6.7 \%)$ & $5(16.7 \%)$ \\
Septum - arcuate uterus & $5(16.7 \%)$ & $9(30 \%)$ & $8(26.7 \%)$ \\
Unsatisfactory result & $7(23.3 \%)$ & $2(6.7 \%)$ & - \\
\hline
\end{tabular}

Table 2. Hysterosalpingographic and hysteroscopic cross comparison of uterine findings.

\begin{tabular}{lcccc}
\hline & HS & HS & HS & HS \\
\cline { 2 - 5 } & Normal & Filling defect (polyp) & Septum - arcuate uterus & Unsatisfactory result \\
\hline $\begin{array}{l}\text { HSG } \\
\text { Normal }\end{array}$ & 11 & 4 & 2 & - \\
HSG & 2 & - & - & - \\
$\begin{array}{l}\text { Filling defect (polyp) } \\
\text { HSG }\end{array}$ & 2 & 1 & 6 & - \\
$\begin{array}{l}\text { Septum - arcuate uterus } \\
\text { HSG }\end{array}$ & 2 & - & - & - \\
$\begin{array}{l}\text { Unsatisfactory result } \\
\text { Total (n) }\end{array}$ & 17 & 5 & 8 & - \\
\hline
\end{tabular}

HS, hysteroscopy; HSG, hysterosalpingography.

terosalpingographic evaluation, it was observed that there was passage through the bilateral fallopian tube in twentyfour women. The evaluation of the remaining six women favored that three had no passage through the right tube, and three through the left tube. Findings regarding the comparative evaluation of tubal passages in women are presented in detail in Table 3.

When the reference method was taken as hysterosalpingography, the specificity of hysteroscopy was determined as $85.71 \%$ (95\% CI: $42.13 \%-99.64 \%)$ and sensitivity was $94.74 \%$ (95\% CI: $85.38 \%-98.9 \%)$, since only the inner mouths of the fallopian tubes can be evaluated in hysteroscopy in the evaluation of the tubal passage. The positive predictive value of hysteroscopy was calculated as 98.18\% (95\% CI: 89.78\%-99.7\%) and the negative predictive value as $66.67 \%$ (95\% CI: $38.96 \%-86.24 \%$ ). The test can give an accurate result with a probability of $93.75 \%$ (95\% CI: $84.76 \%-98.27 \%$ ). When the reference method is taken as hysterosalpingography, the evaluation of the fallopian tubes with office hysteroscopy is statistically significant in terms of the results obtained $(p<0.01)$. And it appears to be a viable method. Table 4 presents the general evaluation of tubal passages obtained by two different evaluation methods.

\section{Discussion}

There are many methods for evaluating tubes. These methods also have many advantages and disadvantages. HSG is the most widely used method in the evaluation of the fallopian tubes and uterine cavity because of its low cost and the fact that it is a procedure that does not require hospitalization. However, of course, the gold standard method is to observe the transition laparoscopically [13].
The swirl created by the water flowing from the sink shows us that the sink drain is open. In some of the women we performed hysteroscopy we noticed that spontaneous bubbles are directed from the inner mouths of the fallopian tubes into the abdomen. Likewise, we observed that the liquid mixed with blood also formed a whirlpool at the same points and directed towards the inside of the abdomen. Inspired by this events, we formed the basis of our work.

Duraker et al. [14] compared laparoscopic chromopertubation and hysterosalpingography in terms of tubal patency. According to their results, while no tubal pathology was observed with L/S in $61.2 \%$ of patients who were diagnosed as unilateral tubal occlusion in $\mathrm{HSG}$, this ratio was $51.2 \%$ in patients with bilateral tubal factor.

A meta-analysis compared hysterosalpingography with laparoscopic chromopertubation, which is the gold standard method for the evaluation of fallopian tubes, and reported the sensitivity of hysterosalpingography to show tubal passage as $65 \%$ and specificity as $83 \%[15,16]$. While this is the case, although the power to evaluate the passage through the fallopian tubes is not as much as laparoscopic chromopertubation, it is considered that it does not change the planned treatment methods by 95\% [17]. And therefore the reference test for evaluating the fallopian tubes in our study was accepted as hysterosalpingography and the statistical evaluation was performed. This situation of course creates a limitation for our study and affects the generalizability of the results we obtain. Another limiting factor may be the sample size of the study. On the other hand, although the power analyzes required for the number of samples were performed and the lowest number of cases required to be evaluated was determined as twenty, thirty cases (sixty tube) were included in the study, but this num- 
Table 3. Hysterosalpingographic and hysteroscopic cross evaluation and comparison of the tubal patency.

\begin{tabular}{lccccc}
\hline & HS & HS & HS & HS & Total \\
\cline { 2 - 6 } & $(\mathrm{R}+)(\mathrm{L}+)$ & $\mathrm{R}(+)(\mathrm{L}-)$ & $(\mathrm{R}-)(\mathrm{L}+)$ & $(\mathrm{R}-)(\mathrm{L}-)$ & $(\mathrm{n})(\mathrm{n})$ \\
\hline $\begin{array}{l}\mathrm{HSG} \\
(\mathrm{R}+)(\mathrm{L}+)\end{array}$ & $(21)(21)$ & $(2)(2)$ & $(1)(1)$ & $(-)(-)$ & $(24)(24)$ \\
HSG & & & & & \\
$(\mathrm{R}+)(\mathrm{L}-)$ & $(-)(-)$ & $(3)(3)$ & $(-)(-)$ & $(-)(-)$ & $(3)(3)$ \\
HSG & & & & & \\
$(\mathrm{R}-)(\mathrm{L}+)$ & $(1)(1)$ & $(-)(-)$ & $(2)(2)$ & $(-)(-)$ & $(3)(3)$ \\
$\begin{array}{l}\text { HSG } \\
(\mathrm{R}-)(\mathrm{L}-)\end{array}$ & $(-)(-)$ & $(-)(-)$ & $(-)(-)$ & $(-)(-)$ & $(-)(-)$ \\
Total (n) (n) & $(22)(22)$ & $(5)(5)$ & $(3)(3)$ & $(-)(-)$ & $(30)(30)$ \\
\hline
\end{tabular}

Table 4. Statistical evaluation of hysteroscopy and hysterosalpingography over each other in the evaluation of tubal patency.

\begin{tabular}{|c|c|c|c|c|c|c|c|c|c|}
\hline \multirow{2}{*}{ HSG } & \multicolumn{2}{|c|}{ HS } & \multirow{2}{*}{ Total } & \multicolumn{5}{|c|}{ HS } & $p$ value \\
\hline & Tubal passage $(+)$ & Tubal passage (-) & & Specificity & Sensitivity & $\begin{array}{c}\text { Positive } \\
\text { predictive value }\end{array}$ & $\begin{array}{c}\text { Negative } \\
\text { predictive value }\end{array}$ & Accuracy & (Fischer exact $<0.01$ ) \\
\hline Tubal passage $(+)$ & $\begin{array}{c}51 \\
(85 \%)\end{array}$ & $\begin{array}{c}3 \\
(5 \%)\end{array}$ & $\begin{array}{l}54 / 60 \\
(90 \%)\end{array}$ & & & & & & \\
\hline Tubal passage (-) & $\begin{array}{c}1 \\
(1.7 \%)\end{array}$ & $\begin{array}{c}5 \\
(8.3 \%)\end{array}$ & $\begin{array}{l}6 / 60 \\
(10 \%)\end{array}$ & $85.71 \%$ & $94.74 \%$ & $98.18 \%$ & $66.67 \%$ & $93.75 \%$ & 0.00001 \\
\hline Total & $\begin{array}{c}52 / 60 \\
(86.7 \%)\end{array}$ & $\begin{array}{c}8 / 60 \\
(13.3 \%)\end{array}$ & $\begin{array}{c}60 / 60 \\
(100 \%)\end{array}$ & & & & & & \\
\hline
\end{tabular}

ber may also be insufficient in terms of generalizing the results. Alternative methods to HSG have been tried to be developed for years. However, studies on the evaluation of tubal patency by hysteroscopy are very limited in the literature $[18,19]$. It is a known fact that hysteroscopy is the gold standard method for detecting lesions related to the uterine cavity [20]. Therefore, in our study, we did not evaluate the power of hysteroscopy to detect lesions in the uterine cavity. We presented the results we obtained on this subject as a table in order to make comparisons only and to better understand the superiority of the method by providing subject integrity. In our study, just like the others, the superiority of hysteroscopy was once again emphasized because it also enables the treatment of detected pathologies [20,21]. Parry et al. [22] measured the ability of office hysteroscopy to evaluate the tubal patency using the Parryscope technique. They reported that with the use of small-caliber ( $<3 \mathrm{~mm}$ ) flexible hysteroscopes, it is possible to obtain very high accuracy results by avoiding excessive distension and preventing the occurrence of spasm. They found that office hysteroscopy has $98.3 \%-100 \%$ sensitivity and $83.7 \%$ specificity for tube occlusion when applied according to the procedure. They suggested that office hysteroscopy can evaluate the patency of the tube accurately and quickly with this method. And they reported that it is a promising technique for evaluating fertility rather than HSG [22]. Although we could not compare hysteroscopy with laparoscopic chromopertubation, which is the gold standard method [23], our results show that hysteroscopy provides similar and even better results with hysterosalpingography in the evaluation of tubes. On top of that, considering the obvious advantage of hysteroscopy over hysterosalpingography, as shown in previous studies, in the evaluation of the uterine cavity, its benefit is further increased.

\section{Conclusions}

Considering the results we obtained, it is seen that hysteroscopy has advantages and disadvantages compared to hysterosalpingography in the evaluation of infertile women. We still think that more research should be done on which method will be more useful in the evaluation of infertile women.

\section{Author contributions}

BAY: Materials, Data Collection and/or Processing. EP, Literature Review, Writer, Critical Review. MAU, Conception, Design, Supervision. All authors participated in data interpretation, statement and approved the final version of the manuscript.

\section{Ethics approval and consent to participate}

All subjects gave their informed consent for inclusion before they participated in the study. The study was conducted in accordance with the Declaration of Helsinki, and the protocol was approved by the Ethics Committee of Canakkale 18 Mart University Scientific Research Ethics Committee: 15.11.2017 / 2017-18 (2011-KAEK-27/2017E.98469). 


\section{Acknowledgment}

Thanks to all the peer reviewers for their opinions and suggestions.

\section{Funding}

This research received no external funding.

\section{Conflict of interest}

The authors declare no conflict of interest.

\section{References}

[1] El-Toukhy TA. Unexplained subfertility: a pragmatic approach. Journal of Obstetrics and Gynaecology. 2002; 22: 239-242.

[2] Serafini P, Batzofin J. Diagnosis of female infertility. A comprehensive approach. Journal of Reproductive Medicine. 1989: 34: 29-40.

[3] Killick SR. Fallopian tube patency testing. In Ledger W, Tan SL, Bahathiq A (eds.) The fallopian tube in infertility and IVF practice (pp. 70-84). New York, USA: Cambridge University Press. 2010.

[4] Godinjak Z, Idrizbegović E. Should diagnostic hysteroscopy be a routine procedure during diagnostic laparoscopy in infertile women? Bosnian Journal of Basic Medical Sciences. 2008; 8: 44-47.

[5] Hager M, Simek IM, Promberger R, Ott J. The Role of Diagnostic Hysteroscopy in the Evaluation of Fallopian Tube Patency: a Short Review. Geburtshilfe Und Frauenheilkunde. 2019; 79: 483-486.

[6] Luciano DE, Exacoustos C, Luciano AA. Contrast Ultrasonography for Tubal Patency. Journal of Minimally Invasive Gynecology. 2014; 21: 994-998.

[7] Exacoustos C, Zupi E, Carusotti C, Lanzi G, Marconi D, Arduini D. Hysterosalpingo-contrast sonography compared with hysterosalpingography and laparoscopic dye pertubation to evaluate tubal patency. Journal of the American Association of Gynecologic Laparoscopists. 2003; 10: 367-372.

[8] Yücel B, Demirel E, Kelekci S, Shawki O. Hysteroscopic evaluation of tubal peristaltic dysfunction in unexplained infertility. Journal of Obstetrics and Gynaecology. 2018; 38: 511-515.

[9] Török P, Major T. Accuracy of assessment of tubal patency with selective pertubation at office hysteroscopy compared with laparoscopy in infertile women. Journal of Minimally Invasive Gynecology. 2012; 19: 627-630.

[10] Habibaj J, Kosova H, Bilali S, Bilali V, Qama D. Comparison between transvaginal sonography after diagnostic hysteroscopy and laparoscopic chromopertubation for the assessment of tubal patency in infertile women. Journal of Clinical Ultrasound. 2012; 40: 68-73.

[11] Salazar CA, Isaacson KB. Office Operative Hysteroscopy: an
Update. Journal of Minimally Invasive Gynecology. 2018; 25: 199-208.

[12] Parry JP, Riche D, Aldred J, Isaacs J, Lutz E, Butler V, et al. Proximal Tubal Patency Demonstrated through Air Infusion during Flexible Office Hysteroscopy is Predictive of whole Tubal Patency. Journal of Minimally Invasive Gynecology. 2017; 24: 646-652.

[13] Pek E, Canbey Göret C, Hacivelioğlu S, Adam G, Ünsal MA. The immunohistochemical and histologic effects of contrast medium on uterus, fallopian tubes and ovaries, given during hysterosalpingography: rat study. Journal of the Turkish-German Gynecological Association. 2020; 21: 243-254.

[14] Duraker R, Demir B, Dilbaz B, Akkurt O, Kocak M, Tasci Y, Goktolga U. Comparisions of hysterosalpingography and laparoscopy results in the diagnosis of tubal occlusion. Turkish Journal of Obstetrics and Gynecology. 2011: 8: 40-43.

[15] Swart P, Mol BW, van der Veen F, van Beurden M, Redekop WK, Bossuyt PM. The accuracy of hysterosalpingography in the diagnosis of tubal pathology: a meta-analysis. Fertility and Sterility. 1995; 64: 486-491.

[16] Aeby TC, Huang T, Nakayama RT. The effect of peritoneal fluid from patients with endometriosis on human sperm function in vitro. American Journal of Obstetrics and Gynecology. 1996; 174: $1779-1775$.

[17] Lavy Y, Lev-Sagie A, Holtzer H, Revel A, Hurwitz A. Should laparoscopy be a mandatory component of the infertility evaluation in infertile women with normal hysterosalpingogram or suspected unilateral distal tubal pathology? European Journal of Obstetrics \& Gynecology and Reproductive Biology. 2004; 114: 64-68.

[18] Vitale SG, Carugno J, Riemma G, Török P, Cianci S, De Franciscis $\mathrm{P}$, et al. Hysteroscopy for Assessing Fallopian Tubal Obstruction: a Systematic Review and Diagnostic Test Accuracy Metaanalysis. Journal of Minimally Invasive Gynecology. 2021; 28: 769-778.

[19] Hager M, Ott J, Holzer I, Seemann R, Kurz C, Parry JP. Hysteroscopic Assessment of Tubal Patency: a Randomized Comparison between the Flow and Parryscope Techniques. Journal of Minimally Invasive Gynecology. 2020; 27: 1552-1557.e1.

[20] Wadhwa L, Rani P, Bhatia P. Comparative Prospective Study of Hysterosalpingography and Hysteroscopy in Infertile Women. Journal of Human Reproductive Sciences. 2017; 10: 73-78.

[21] Malhotra V, Chauhan MB, Lakra P, Sangwan R. Hysterosalpingography vs Hysteroscopy: Role in Assessment of Uterine Factor during Infertility Workup. International Journal of Infertility \& Fetal Medicine. 2013; 4: 79-82.

[22] Parry JP, Riche D, Rushing J, Linton B, Butler V, Lindheim SR. Performing the Parryscope technique gently for office tubal patency assessment. Fertility and Sterility. 2017; 108: 718.

[23] Ott J, Hager M, Nouri K, Marschalek J, Kurz C. Assessment of Tubal Patency: a Prospective Comparison of Diagnostic Hysteroscopy and Laparoscopic Chromopertubation. Journal of Minimally Invasive Gynecology. 2020; 27: 135-140. 